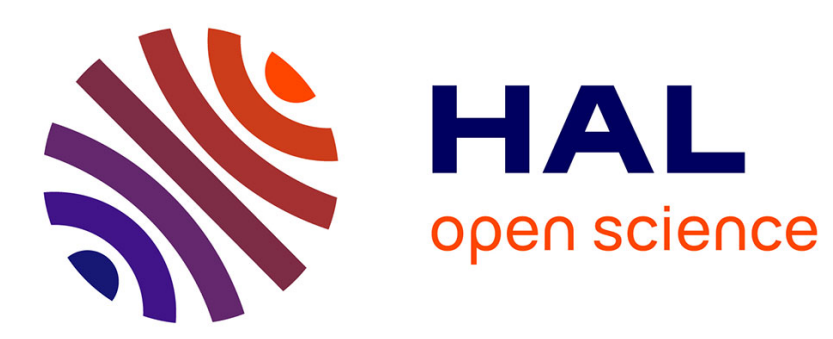

\title{
Time-resolved evolution of plasma parameters in a plasma immersion ion implantation source
}

J. Moreno, A. Khodaee, D. Okerstrom, M. P Bradley, Lénaïc Couëdel

\section{To cite this version:}

J. Moreno, A. Khodaee, D. Okerstrom, M. P Bradley, Lénaïc Couëdel. Time-resolved evolution of plasma parameters in a plasma immersion ion implantation source. Physics of Plasmas, 2021, 28 (12), pp.123523. 10.1063/5.0063610 . hal-03517375

\section{HAL Id: hal-03517375 \\ https://hal.science/hal-03517375}

Submitted on 7 Jan 2022

HAL is a multi-disciplinary open access archive for the deposit and dissemination of scientific research documents, whether they are published or not. The documents may come from teaching and research institutions in France or abroad, or from public or private research centers.
L'archive ouverte pluridisciplinaire $\mathbf{H A L}$, est destinée au dépôt et à la diffusion de documents scientifiques de niveau recherche, publiés ou non, émanant des établissements d'enseignement et de recherche français ou étrangers, des laboratoires publics ou privés. 


\title{
Time-resolved evolution of plasma parameters in a plasma immersion ion implantation source
}

\author{
J. Moreno, ${ }^{1, *}$ A. Khodaee, ${ }^{1}$ D. Okerstrom, ${ }^{1}$ M. P. Bradley, ${ }^{1, \dagger}$ and L. Couëdel ${ }^{1,2}$, 周 \\ ${ }^{1}$ Department of Physics and Engineering Physics, \\ University of Saskatchewan, Saskatoon, SK S7N 5E2, Canada \\ ${ }^{2}$ Aix-Marseille Université, CNRS, PIIM, UMR 7345, \\ Centre Scientifique de Saint Jérôme, case 241, 13397 Marseille Cedex 20, France
}

(Dated: January 7, 2022)

\begin{abstract}
The origin and nature of perturbations induced by a high-voltage pulse on plasma parameters and their relationship to operating conditions (power and pressure) in an argon inductively coupled radio-frequency plasma device is explored. The plasma parameters are measured with two radiofrequency compensated Langmuir probes positioned either vertically above the pulsing target or horizontally along the diameter of the chamber, in the same axial plane as the target and same distance from the RF antenna. Fluctuations are observed in electron density $n_{e}$, temperature $T_{e}$ and plasma potential $V_{p l}$ following negative polarity high voltage pulses, and propagate deep in the plasma and well after the end of the pulse. Time-resolved data results indicate that the perturbations are significantly dampened at higher power, as well as when closer to the plasma RF coil. The perturbation amplitudes depart significantly from steady state values when the pulse amplitude exceeds $2.0 \mathrm{kV}$, and increase with increasing pulse amplitude. Perturbation amplitudes are also higher for target materials having larger secondary electron yield. Our experimental results suggest that the underlying mechanism of this perturbation could be plasma heating driven by damping of a beam-plasma instability as a result of a beam of secondary electrons emitted by the target streaming into the plasma.
\end{abstract}

\section{INTRODUCTION}

Plasma immersion ion implantation (PIII) is a materials processing technology with many applications such as modification of material surfaces [1], fabrication of microelectronic and optoelectronic devices [2 7, and the synthesis of novel materials [8, 9]. In PIII, the target to be processed is immersed in a plasma, which acts as a source of ions. The ions are implanted by applying a negativepolarity high voltage (NPHV) bias to the target. This high voltage is pulsed because very high power requirements typically exceeds the capacity of a DC supply (in terms of power and/or current intensity). Furthermore, a short duty cycle pulsed bias allows cooling to avoid target damage. PIII provides high-fluence implantation over large areas making it advantageous for ion implantation applications such as large diameter semiconductor wafers, solar cells, flat panel displays, etc. Furthermore, it is an adaptable process that accommodates high and low plasma densities $\left(10^{8}\right.$ to $\left.10^{12} \mathrm{~cm}^{-3}\right)$, a wide ion energy range ( $1 \mathrm{keV}$ to $300 \mathrm{keV})$ and a broad range of operating pressures $(<1 \mathrm{mTorr}$ to $>100$ Torr $)$ [10]. PIII can effectively processes irregularly shaped objects as a result of the conformal sheath that naturally develops between the bulk plasma and any plasma-facing surface (see Ch. 4 of Ref. [10]). Ion implantation can occur either by continually biasing the target, as in Capacitively Coupled Plasma (CCP) discharges [11, or by placing a

\footnotetext{
* jtm220@usask.ca

$\dagger$ michael.bradley@usask.ca

$\ddagger$ lenaic.couedel@usask.ca
}

target on a metal stand and applying NPHV pulses, as in Inductively Coupled Plasma (ICP) discharges [11.

PIII processes planning for device fabrication requires predictability of the ion fluence per pulse. A preliminary model of the PIII process was first proposed by Conrad [12] and was further developed by Lieberman [13]. This model (referred to later as the Lieberman's model) enables calculation of the ion fluence delivered to the target during a high voltage pulse, in terms of the bulk plasma density and electron temperature. The model captures the basic physics of high voltage sheath expansion by solving Poisson's equation during the NPHV pulse. First, the NPHV pulse drives electrons away from the region adjacent to the target. Solving Poisson's equation for the transient case of stationary ions results in an ion matrix sheath with thickness $S_{M}$ given by:

$$
S_{M}=\left(\frac{2 \epsilon_{0}\left|V_{s}\right|}{e n_{s}}\right)^{1 / 2}=\lambda_{D}\left(\frac{2\left|V_{s}\right|}{T_{e}}\right)^{1 / 2} .
$$

Here, $\epsilon_{0}$ is the vacuum permittivity, $e$ is the elementary charge, $n_{s}$ is the plasma density at the sheath edge (normally taken to be $n_{s}=0.61 n_{0}$, where $n_{0}$ is the bulk plasma density), and $\left|V_{s}\right|$ is the amplitude of the NPHV pulse. $\lambda_{D}$ is the Debye length, defined as $\lambda_{D}=\sqrt{\epsilon_{0} T_{e} / n_{0} e}$, where $T_{e}$ is given in $\mathrm{eV}$. The matrix sheath is not a steady state solution for a biased target in a plasma, since the plasma ions are mobile [13. As ions accelerate through the sheath and are implanted into the target, more ions enter from the bulk plasma, and the sheath expands. Lieberman's model then solves Poisson's equation for thermal electrons and ballistic ions leading to an ordinary differential equation for the sheath evolu- 
tion and a sheath length that lasts until the end of the pulse. The steady-state high-voltage sheath then obeys the Child-Langmuir law for space-charge limited current with a Child law sheath width $S_{C}$ given by [13:

$$
S_{C}=\frac{\sqrt{2}}{3} \lambda_{D}\left(\frac{2\left|V_{s}\right|}{T_{e}}\right)^{3 / 4} .
$$

For low-temperature, low-pressure plasmas used in plasma processing, the matrix sheath is in the range of a few millimetres and Child law sheath lengths are on the order of $\sim 1 \mathrm{~cm}$. The model assumes constant bulk plasma density and bulk electron temperature. Measured values of steady state plasma parameters can be used to calculate the ion fluence delivered to the target; such calculations are very useful for process planning in industrial ion implantation applications. However, while the Lieberman model does capture the basic physics of the PIII process, it neglects important effects, ultimately reducing its accuracy. A principle shortcoming of this model is that, since the target is immersed in the plasma, the NPHV pulse and the bulk plasma are not isolated but rather have a complex, non-linear relationship. The NPHV pulse is known to be highly perturbative; in fact, for different systems it may be used to ignite a plasma itself without an RF power source, as is the case in pulsed plasma thrusters or high-powered impulse magnetron sputtering [14, 15]. Thus, steady and spatially uniform bulk plasma parameters may not be a valid assumption. Likewise, the sheath that accelerates ions to the target is assumed to consistently have the same structure and physical characteristics (density, potential drop and spatial variations) for each pulsed NPHV deposition event 13. Furthermore, secondary electrons that are ejected by ion bombardment during each NPHV pulse accelerate through the sheath back into the bulk plasma and may disturb it [16. There are numerous extensions to the model which consider finite pulse rise times [17, analytical and numerical models for an expanding sheath edge for multiple pulses [18, 19, as well as aspects of the physics of a collisional sheath [20]. Particle-in-Cell simulations and Monte Carlo numerical solutions are commonly used to study time evolution of HV sheaths [19, 21, 22]. These methods have shown that, due to a finite plasma recovery time, process efficiency for optimal output yield is most affected by pulse repetition rate [23, 24]: the time between pulses must be long enough such that the plasma returns to steady state as well as adhering to target heating constraints [10.

Various authors have worked on improving PIII models to give better process predictability (see Ref. 25-27]). Chung et al. 28 30 and Briehl et al. 31] also conducted a series of experiments focused on studying these nonlinear effects in non-uniform, low-pressure argon plasmas. A simple, linear relationship between plasma recovery time and $S_{C}$ was derived, however it held limited applicability due to neglecting realistic plasma conditions such as non-uniformity, ion drift and a non-constant density of replenishing ions from the bulk. These studies utilized Langmuir probes positioned near the pulsing target to measure the velocity of a "rarefaction wave" that moves with the expanding sheath edge during a NPHV pulse. It was determined that the rarefaction wave separates from the sheath edge and propagates into the plasma at velocities below the Bohm velocity [28]. Cluggish et al. studied large-scale operations involving multiple workpieces in a single ICP chamber and found that sheaths that overlap do not maintain their predicted evolution and lengths [25. Another study by Cluggish et al. found that, in a CCP discharge, secondary electrons streaming into the plasma increase plasma density via resonant energy deposition 32. This phenomenon has been documented in other studies (see Refs. 16, 33), however its impact on the predictive model for PIII processing has yet to be established.

Recent work performed at the University of Saskatchewan revealed perturbations in plasma parameters - plasma density $n_{e}$, electron temperature $T_{e}$ and plasma potential $V_{p l}$ - measured several microseconds after the NPHV pulse and tens of centimetres away from the pulsing target, indicating that the effects of the NPHV pulses extend beyond the duration of the pulse and the length of $S_{C}$ and $S_{M}$ [26. The present research focuses on isolating each individual operating parameter - power, pressure, NPHV pulse amplitude $\left(\left|\Delta V_{s}\right|\right)$ and target material - in order to quantify its effect on the amplitude, depth and time dependence of the NPHV pulse induced perturbations. Additionally, different pulsing target configurations with respect to the plasma source, as well as different target materials, that can alter the amplitude of the perturbation are investigated. Experimental results suggest that the perturbations may be driven by a beam-plasma instability, which is a theory consistent with Refs. [16, 32, 33]. The article is organized as follows: the experimental set-up is described in Sec. 2, while Sec. 3 details the results of the perturbation studies. Finally, the results are discussed in Sec. 4.

\section{EXPERIMENTAL SET-UP}

\section{A. Plasma Chamber}

Fig. 1 is a schematic diagram of the experimental system. The plasma source is an ICP source designed and manufactured by Plasmionique, Inc 34. The vacuum chamber is a stainless steel cylinder (inner diameter of $29.8 \mathrm{~cm}$, height of $46.0 \mathrm{~cm}$ ). The pumping system is composed of an Alcatel Adixen 2010SD Pascal Dual Stage Rotary Vane Vacuum roughing pump that pumps the chamber down to $\sim 1$ mTorr. An air-cooled Leybold turbo-molecular high vacuum pump then establishes a base pressure of $\sim 1 \mu$ Torr before experiments are conducted. An MKS type M100B mass flow controller allows gas flow into the chamber from $1-100 \mathrm{sccm}$ to establish 
a working pressure in the range of 1-100 mTorr. An RF generator operating at $13.56 \mathrm{MHz}$ provides up to $600 \mathrm{~W}$ of input power to a water-cooled, planar coil, RF antenna separated from the chamber by a quartz window. The power supply impedance is matched using a Match Pro CPM-1000 matching network. The present experiments are performed with argon gas and operate at pressures that vary from $1.5-25$ mTorr, flowing at $5 \mathrm{sccm}$, with input power in the range $120-200 \mathrm{~W}$.

The target sits on disk-shaped stainless steel support (area of $36.45 \mathrm{~cm}^{2}$ ). It may be positioned at the bottom on the centre axis of the chamber (vertical configuration), or installed in any one of the side ports along the circumference of the chamber (horizontal configuration). For these experiments the pulses are delivered with a fixed frequency of $250 \mathrm{~Hz}$, and the pulse duration is fixed at 5 $\mu \mathrm{s}$. In the experiment described herein, the NPHV pulse amplitude is varied from 1 to $4 \mathrm{kV}$ in $0.5 \mathrm{kV}$ steps.

\section{B. Langmuir Probe}

The plasma parameters - electron and ion density $n_{e}$ and $n_{i}, T_{e}$, and $V_{p l}$ - are measured by two cylindrical Langmuir probes (LP). The probe design is based on a model for RF-driven plasmas made by Takashi et al. 35. Measurements of the plasma parameters are conducted with an RF-compensated, cylindrical-tip LP. The tips are tungsten wire $1.0 \mathrm{~mm}$ in diameter and $6.37 \mathrm{~mm}$ in length. The probe tip diameter was chosen large enough to draw sufficient current in the density depletion wave propagating through the plasma. The probe is equipped with four inductors in series with resonant frequencies at the first two harmonics of the RF coil, $13.56 \mathrm{MHz}$ and 27.12 MHz. These act as bandpass filters that reduce the effects of RF fluctuations in the plasma potential leading to inaccurately high electron temperature [36. The reference electrode (a copper ring) coupled to the probe tip through a $18 \mathrm{nF}$ capacitor further reduces the influence of RF fluctuations and improves the accuracy of the electron temperature readings [37. In the "vertical configuration", the probes' tips are positioned on the vacuum vessel central axis at $8.7 \mathrm{~cm}$ and $21.7 \mathrm{~cm}$ above the pulsing target, respectively (see Fig. 11 )). Note that the cylindrical tips are perpendicular to the chamber axis. In the "horizontal configuration", the pulsing target and two probes are all positioned at the same distance relative to the bottom of the chamber (and the RF antenna) and aligned along the diameter. Probe $1\left(p_{1}\right)$ is positioned $\left(L_{1}=\right) 6.25 \mathrm{~cm}$ away from the target and probe 2 $\left(p_{2}\right)$ is positioned $\left(L_{1}+L_{2}=\right) 10.275 \mathrm{~cm}$ away from the target as seen in Fig. 1 $\mathrm{b}$ ).

The time-resolved measurements were taken by stepping the probes' bias potential from $-40 \mathrm{~V}$ to $60 \mathrm{~V}$ and recording the probes' current, DC bias, and the NPHV pulse waveform. All four signals, including two LP signals, were output to an oscilloscope (Siglent SDS1104X). The time-resolved Current-Voltage (IV) curves were con- structed by first centring the oscilloscope's $28 \mu$ s time window on the NPHV pulse to record the pulse waveform, the current drawn from the two LP's and the DC voltage applied to the probes. Then, IV curves were built at each time step (i.e. every $2 \mathrm{~ns}$ across the $28 \mu$ s window) from the incrementally increased applied potential, resulting in 14,000 IV curves. The waveform of the probe current during the pulse event was averaged 16 times to reduce noise.

The plasma parameters are calculated from the IV curves using the standard Langmuir analysis [13]. This method is a semi-analytic approach that works well with electrons following a Maxwellian distribution, which has been found to be a reasonable assumption for ICP's in this pressure regime [38. Godyak et al. studied electron distribution functions measured by LP's in detail [39. They investigated electron energy probability function (EEPF) distributions with respect to a broad range of ICP power, pressure, and RF power frequencies. They found that at a driving frequency of $13.56 \mathrm{MHz}$, the EEPF evolves from a bi-Maxwellian distribution at 12.5 $\mathrm{W}$ input power to fully Maxwellian one at $200 \mathrm{~W}$, at a pressure of $10 \mathrm{~m}$ Torr of pressure. This is due to an increase in anomalous collisionless electron heating that enables Maxwellization of the EEPF. Since our operating power and pressure conditions are similar to that of Ref. [39, it is reasonable to assert that the electron distribution functions in our experiment are similarly near Maxwellian.

Preliminary work showed that results from the Langmuir method slightly overestimated density and temperature with respect to theoretical predictions [26]. All compared analysis methods (i.e. OML, ABR, and Druyvesteyn) gave results within an order of magnitude, and often within error bars of each other. The Langmuir analysis is also the least computationally demanding, an important characteristic when processing 40,000 IV curves. Fig. 2 shows a representative Electron Energy Distribution Function (EEDF) measured by a LP in the top port during steady state operation (i.e. no NPHV pulse). This confirms that while not an exact Maxwellian distribution, it is close enough to justify this method, given the other constraints facing this experiment. Of course, further deviation from a Maxwellian distribution may occur during the NPHV pulse. However, given the limiting factor of the number of voltage points and computing power for the vast number of IV curves, the Langmuir analysis was the only practical method to obtain time-resolved plasma parameters. Additionally, given the inherent noise, any improvements to accuracy would likely be overshadowed by the noise induced with the second derivative when attempting the Druyvestein procedure. Furthermore, this report focuses on trends in plasma parameters extracted from LP IV curves, thus systematic error will have a negligible impact on the results of interest. 


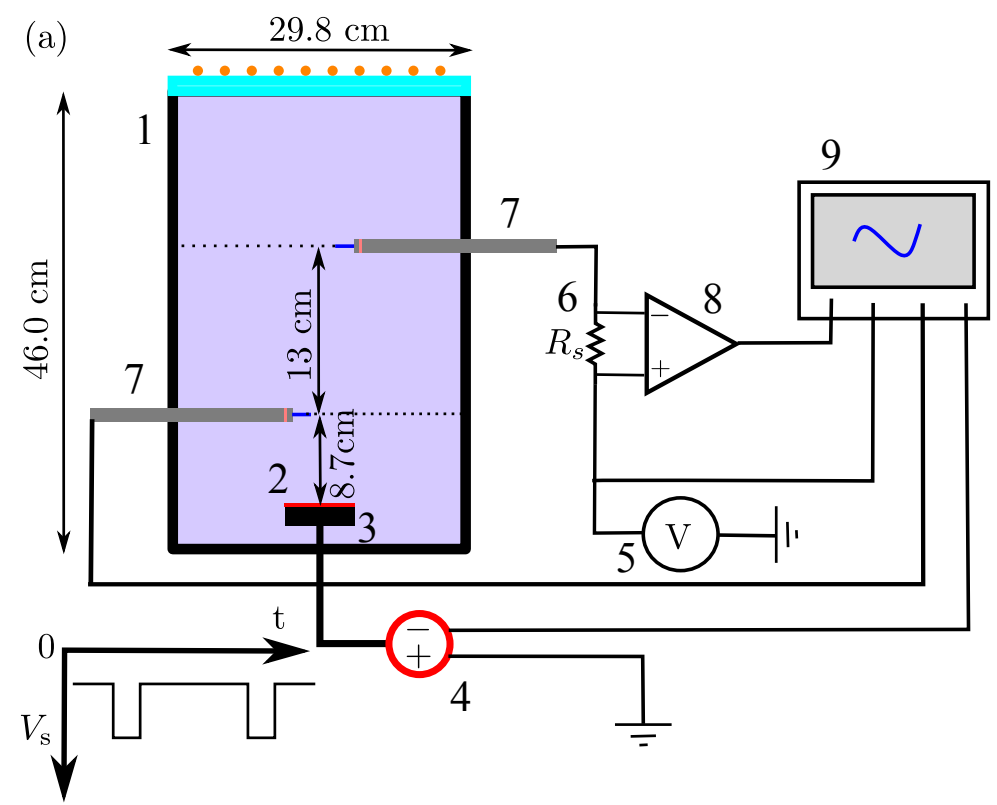

(b)

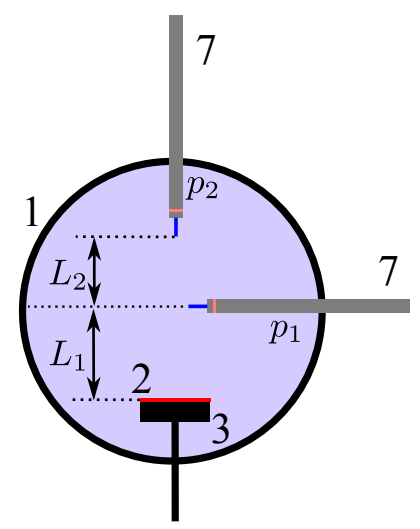

FIG. 1. (Colour online) Schematic of the experimental set up in the (a) vertical configuration and (b) horizontal configuration. (1) ICP plasma chamber; (2) target substrate (silicon or stainless steel); (3) substrate holder; (4) NPHV pulsing target; (5) probe adjustable DC voltage supply; (6) probe sense resistor; (7) RF-compensated Langmuir probe; (8) Operational amplifier; (9) oscilloscope. In the horizontal configuration the probe in front of the target is labelled probe $1\left(p_{1}\right)$ and the probe on the side is labelled probe $2\left(p_{2}\right)$, with $L_{1}=6.25 \mathrm{~cm}$ and $L_{2}=4.025 \mathrm{~cm}$. Both probes and the pulsing target are $10 \mathrm{~cm}$ below the source.

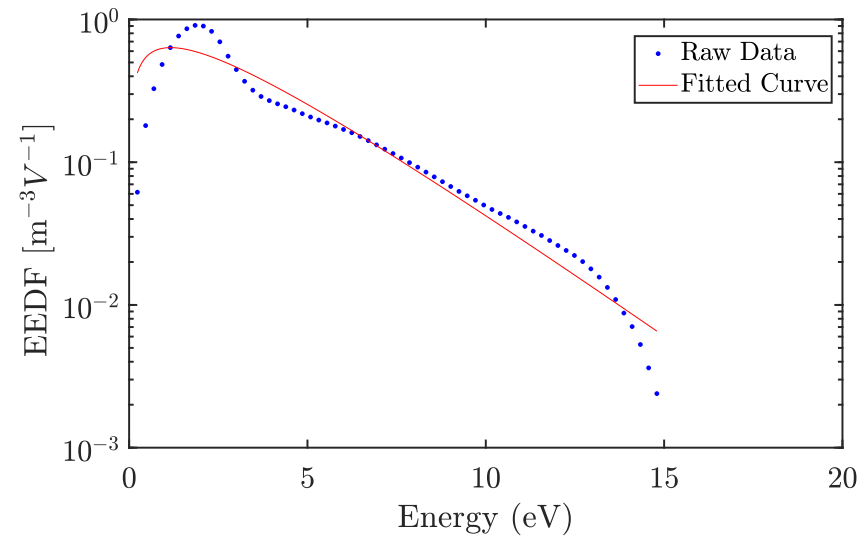

FIG. 2. The Electron Energy Distribution Function (EEDF) on a semi-log plot, taken by the Langmuir probe in the top port. This was taken for an argon plasma at a pressure of 1.5 mTorr and an input power of $120 \mathrm{~W}$.

\section{RESULTS}

\section{A. Steady State}

As mentioned, the EEDF's are close to a Maxwellian distribution, as seen in Fig. 2. Fig. 3 shows that the radial profile of density and temperature are consistently flat out to $6 \mathrm{~cm}$ from the centre, deviating by about $17.5 \%$ for density and $5 \%$ for temperature. At the centre of the chamber, for input power of $120 \mathrm{~W}$ and pressures ranging from 1.5 to 25 mTorr, the density ranges from $n_{e}=10^{10}$ to $10^{11} \mathrm{~cm}^{-3}$ (see Fig. 4), and the measured electron temperature ranges from $T_{e}=1.5$ to $2.5 \mathrm{eV}$ (Fig. 5). Theoretical electron density values calculated from a global model of a cylindrical ICP described in Ref. 11] are also plotted to confirm the validity of these measurements.

\section{B. Perturbation Studies}

\section{Effects of Power, Pressure and NPHV Pulse Amplitude}

First, the study determines the operating conditions power, pressure and $\left|\Delta V_{s}\right|$ - that result in the greatest perturbation amplitude in plasma parameters, relative to steady state pre-pulse values. Perturbations are calculated by normalizing the plasma parameter value over the $28 \mu$ s time window, e.g. $n_{e}(t)$, to the mean of prepulse values, $n_{e, 0}$.

$$
\Delta n_{e}=\frac{\left|n_{e}(t)-n_{e, 0}\right|}{n_{e, 0}} .
$$

The deviation from this pre-pulse value defines the perturbation amplitude. An example of these results is seen in Fig. 6, and similar calculations are done for $T_{e}$ and $V_{p l}$. The error bars are determined in two steps. First, the 

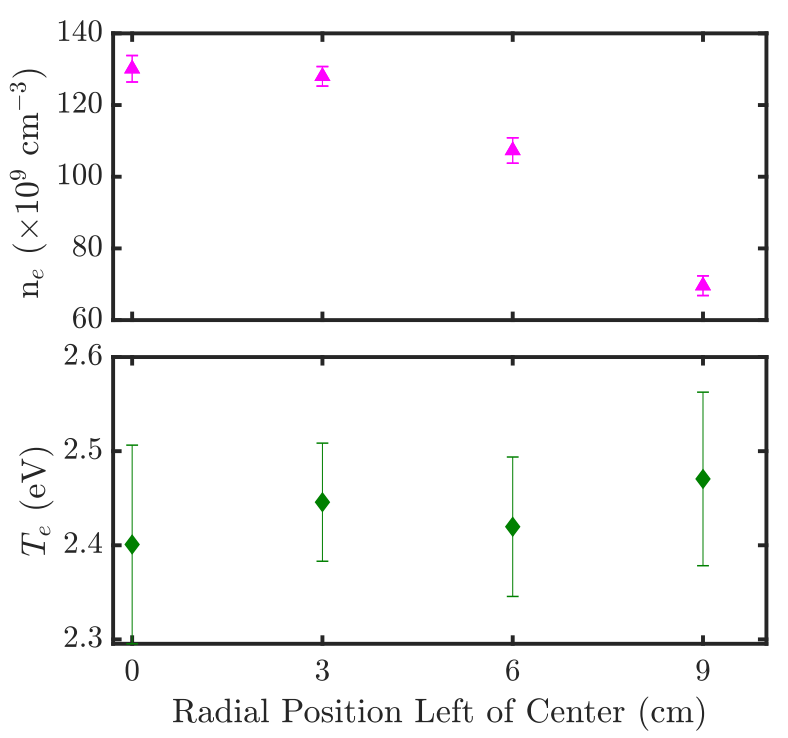

FIG. 3. Radial profile of electron density $n_{e}$ (top) and electron temperature $T_{e}$ (bottom) measured at $1.5 \mathrm{~m}$ Torr and $120 \mathrm{~W}$ input power.

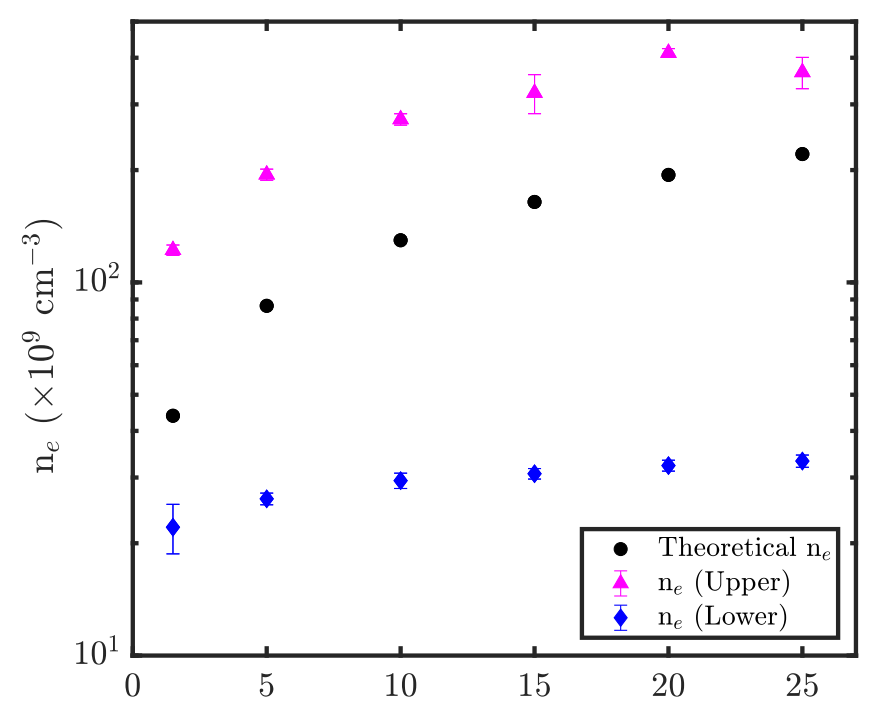

FIG. 4. Electron density across the range of pressures used for these experiments, with $120 \mathrm{~W}$ input power and measured $21.7 \mathrm{~cm}$ from the target (upper position) and $8.7 \mathrm{~cm}$ from the target (lower position) in the plasma chamber. The measured values and their error, shown in pink and blue, are plotted against theoretical values calculated from a global model of ICP plasma generation under equivalent conditions. Note that the range in values in the top port spreads over about $3 \times 10^{11} \mathrm{~cm}^{-3}$, whereas it is only about $1 \times 10^{10} \mathrm{~cm}^{-3}$ in the bottom port.

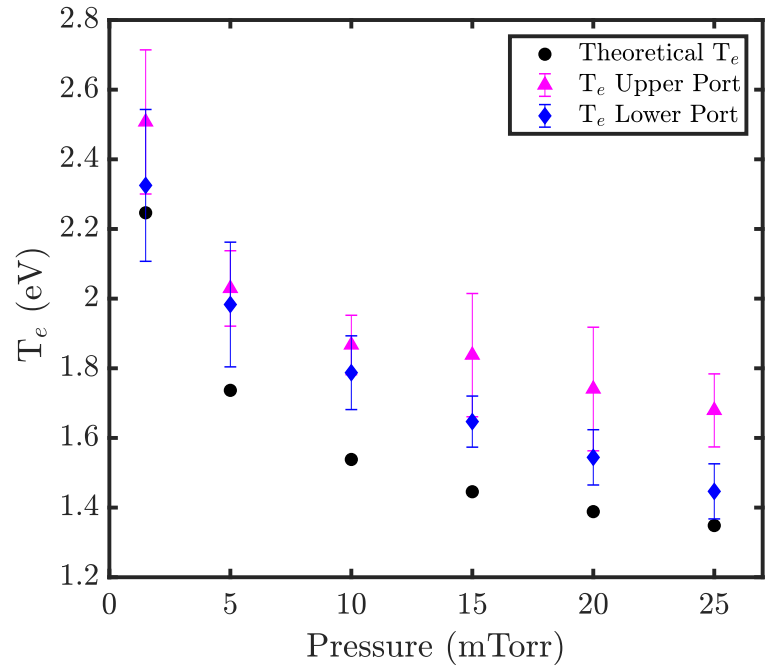

FIG. 5. Electron temperature values across the range of relevant pressures measured in the top port and the bottom port, calculated by taking the inverse of the slope of the transition region (Langmuir method). The measured values and their error, shown in pink and blue, are plotted against theoretical values calculated from a global model of ICP plasma generation under equivalent conditions. Measurements were taken with an input power of $120 \mathrm{~W}$.

error for the time-resolved plasma parameters (the gray shading in Fig. 6) was calculated from fitting functions used for the ion saturation, transition region and electron saturation regions of the IV curve with $95 \%$ confidence intervals, in accordance with the Langmuir method [40. Then, a propagation of error is performed on equation 3 to determine the error bars. It should be noted here that while this error analysis involves the error inherent to the method of data processing and its propagation in the perturbation studies, the analysis does not account for the additional error due to deviations from Maxwellian EEDF. Therefore, the error reported here serves as a lower bound. However, since the study focuses on the time evolution of these perturbations as a function of power and pressure, the accuracy of the absolute values is not of major importance.

Figs. 7 and 8 show that perturbation amplitudes in electron density and plasma potential depend on the input power of the plasma, with increasing power effectively dampening the perturbative effect of the pulse. However, Fig. 9 shows that the electron temperature is weakly dependent on the power and pressure in terms of the perturbation amplitude, and only starts to show a slight departure from pre-pulse values at $\left|\Delta V_{s}\right| \geq 3.5$ $\mathrm{kV}$. 
a)
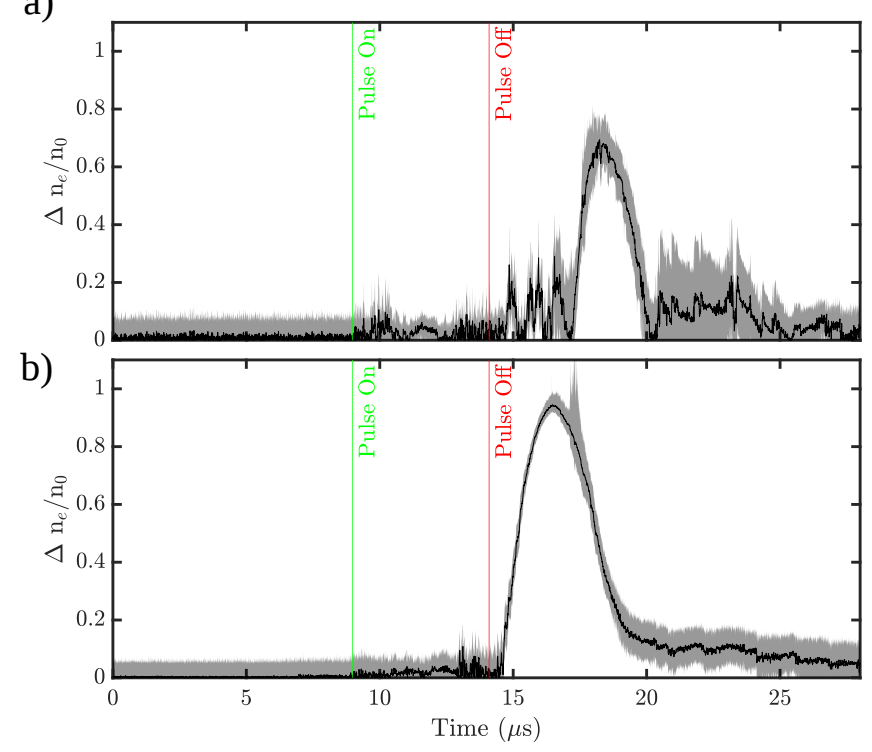

FIG. 6. Electron density perturbations measured by a Langmuir probe at the a) top port, $21.7 \mathrm{~cm}$ from the target and b) the bottom port, $8.7 \mathrm{~cm}$ from the stand. The start and end of the pulse are marked in green and red respectively. The pressure was $1.5 \mathrm{~m}$ Torr, the input power was $120 \mathrm{~W}$ and the pulse amplitude $\left|V_{s}\right|=4.0 \mathrm{kV}$.

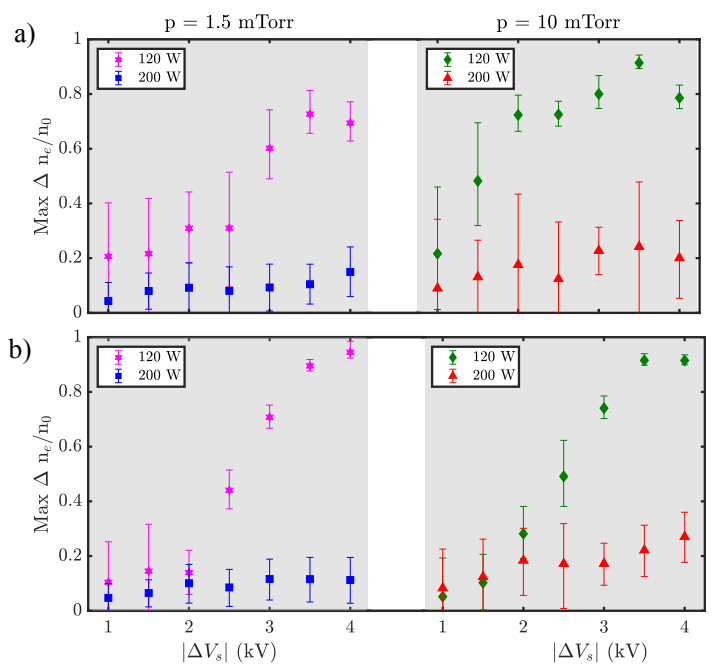

FIG. 7. Electron density maximum perturbation amplitude vs $\left|\Delta V_{s}\right|$ for various power and pressure values, measured a) $21.7 \mathrm{~cm}$ and b) $8.7 \mathrm{~cm}$ from the stand. Note the departure from pre-pulse values $\gtrsim 2.0 \mathrm{kV}$, as well as reduced amplitudes with increasing power.
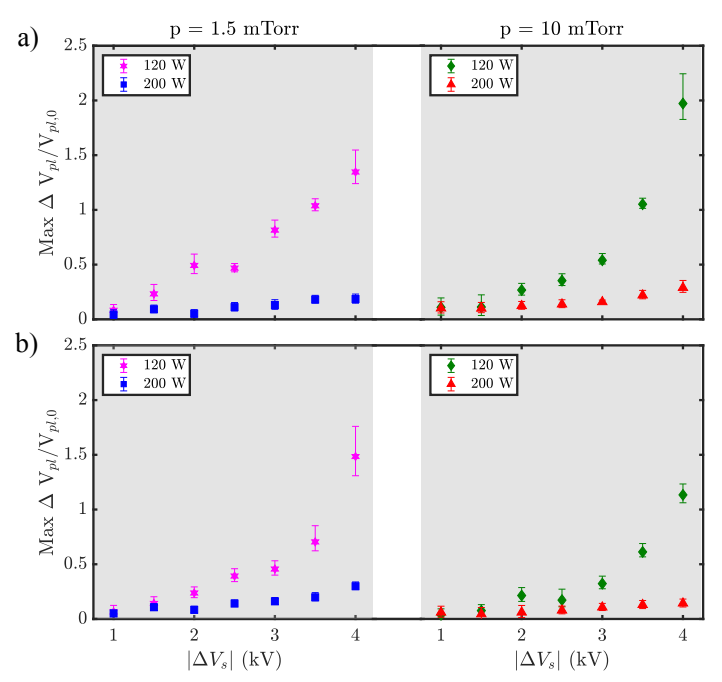

FIG. 8. Plasma potential maximum perturbation amplitude vs $\left|\Delta V_{s}\right|$ for various power and pressure values measured a) $21.7 \mathrm{~cm}$ and b) $8.7 \mathrm{~cm}$ from the stand. Again, there is a clear departure in perturbation amplitude from pre-pulse values $\gtrsim$ $2.0 \mathrm{kV}$ and the pressure dependence exists for this plasma parameter as well.
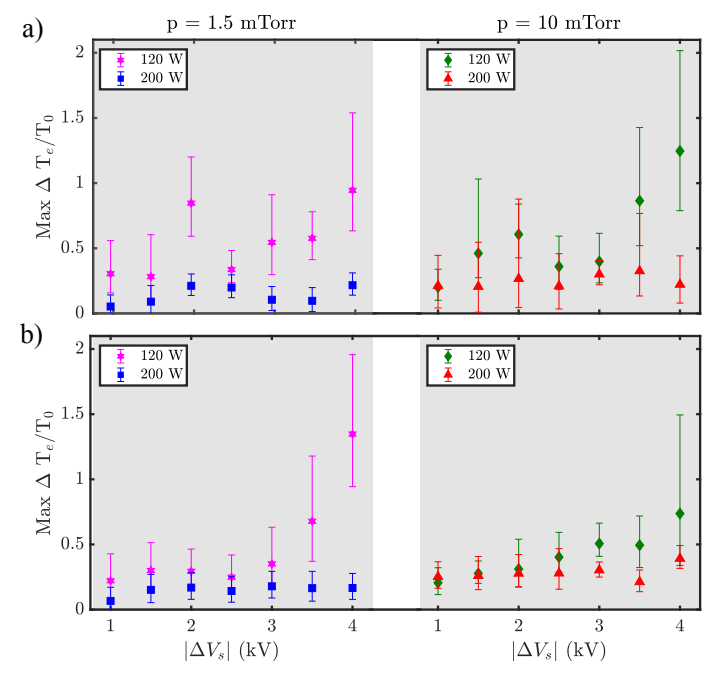

FIG. 9. Electron temperature maximum perturbation amplitude vs $\left|\Delta V_{s}\right|$ for various power and pressure values measured a) $21.7 \mathrm{~cm}$ and b) $8.7 \mathrm{~cm}$ from the stand. Most perturbation amplitudes do not depart significantly from pre-pulse values, and most outliers are in the range of errorbars of pre-pulse values. 


\section{Perturbations and Varying Pressure: Vertical Configuration}

The previous section shows that at a constant power, the perturbation amplitudes trend upward for increasing $\left|\Delta V_{s}\right|$, while increasing the power has the effect of decreasing the perturbation amplitude for any given pressure. The present section now focuses on the effects of $\left|\Delta V_{s}\right|$ on plasma parameter perturbation amplitude for pressure values ranging from $1.5 \mathrm{~m}$ Torr to $25 \mathrm{mTorr}$. As shown in Figs. 10 and 11, perturbations in $n_{e}$ and $V_{p l}$ significantly depart from steady-state, pre-pulse values for NPHV amplitudes $\gtrsim 2.0 \mathrm{kV}$ for all pressures, while $T_{e}$ remains relatively unperturbed across increasing pressures and NPHV amplitudes. This is consistent with the results from the power studies shown in Figs. 7- 9 .

Figs. 10 and 11 show that proximity to the RF coil affects the plasma parameter perturbation amplitudes. Measurements taken close to the RF coil tend to have lower perturbation amplitudes for most pressures, except at 10 mTorr, than measurements taken close to the target and far from the RF coil. At $21.7 \mathrm{~cm}$, the largest perturbation amplitudes are between 1.5 mTorr and 10 mTorr; beyond these pressures, the trends are less discernible and the maximum perturbation amplitude slightly decreases. At $8.7 \mathrm{~cm}$, increasing the pressure does not appreciably affect either the max perturbation amplitude, or the trend in amplitudes across increasing $\left|\Delta V_{s}\right|$. Finally, similar to the results shown in Fig. 9, electron temperature does not show as strong of a dependence on $\left|\Delta V_{s}\right|$ as the one exhibited by density or plasma potential (see Fig. 11).

\section{Perturbations and Varying Pressure: Horizontal Configuration}

While measurements shown in Figs. 10 and 11 provide a quantitative description of the amplitude of the perturbation along the chamber axis, density and temperature gradients from the RF coil to the pulsing target make it difficult to accurately interpret the spatial dynamics and evolution of the perturbation relative to the distance to the pulsing target. The LP's were re-positioned such that both were installed in the upper ports, along with the NPHV pulsing target, as seen in Fig. 1p). In this configuration, the distance from the pulsing target to probe 1 is $L_{1}=6.25 \mathrm{~cm}$, and the distance from probe 2 to the pulsing target is $L_{1}+L_{2}=10.275 \mathrm{~cm}$. The probe tips are centred on the pulsing target.

This configuration enables study of the perturbation with minimal effects from asymmetries in the plasma profiles. Furthermore, the pulsing target is now positioned much closer and oriented in such a way that its surface area is now perpendicular to the source. Both probes are now also equidistant to the $\mathrm{RF}$ coil, $\sim 10 \mathrm{~cm}$. The plasma density perturbations for two difference pressures $(\mathrm{p}=1.5 \mathrm{~m}$ Torr and $\mathrm{p}=10 \mathrm{~m}$ Torr $)$ are plotted in Fig. 12 (vertical configuration in $12 \mathrm{a}$ ), horizontal configuration in $12 \mathrm{p})$ ).

Fig. 12a) isolates the $1.5 \mathrm{~m}$ Torr and $10 \mathrm{mTorr}$ data from Fig. 10 to emphasize the difference in perturbation amplitudes between the top (21.7 $\mathrm{cm}$ from the target) and bottom (8.7 cm from target) LP's. Whereas perturbations measured by the top probe exhibit greater dependence on pressure, bottom probe measurements do not. Conversely, probes in the horizontal configuration (Fig. 12 b)) measure perturbation amplitudes that are not as sensitive to pressure regardless of proximity to the pulsing target, and in general have lower amplitudes than their vertical configuration counterparts. The proximity to the pulsing target has a smaller impact on perturbation amplitude as well, as the points remain within error bars of one another for all $\left|\Delta V_{s}\right|$.

\section{Target Material Dependence}

In order to see whether target secondary electron yield (SEY) coefficients $\left(\gamma_{s}\right)$ affect the plasma perturbations, two different target materials (stainless steel (SS) and silicon $(\mathrm{Si})$ ) were tested. The values of SEY depends on the bombarding ion energy and type. Qin et al. [16] measured $\gamma_{s}$ for Si targets for different ion species. These values follow a scaling law defined as $\gamma_{i}=A \times \sqrt{E_{i}}$, where $\gamma_{i}$ is the SEY of the ion, $A$ is a pre-factor that depends on the ion species (0.8681 for argon), and $E_{i}$ is the ion energy incident on the target, in $\mathrm{keV}$. These values range between 0.93 and 1.92. SEY values for various metals were measured and presented by Phelps et al. 33. For Ar+ ions from 1-5 keV, $\gamma_{s}$ the SEY for the metals ranges between 0.1 and 1.0, depending on metal surface cleanliness.

With up to an order of magnitude difference between SS and Si SEY, an appreciable difference in secondary electron densities measured during the pulse should be detected. The fast secondary electrons manifest as a distinct second slope in the transition region of the IV curves measured during the pulse. A Langmuir analysis was performed on these curves to obtain the density values shown in Fig. 13. Except for the outlier data point at $2.5 \mathrm{kV}$, secondary electron densities are consistently higher for $\mathrm{Si}$ than for $\mathrm{SS}$. Additionally, the $T_{e}$ of secondary electrons emitted during the pulse was measured and is shown to increase with increasing $\left|\Delta V_{s}\right|$ (see Fig. 14. The resulting amplitudes of perturbations in electron density for the two targets are presented in Fig. 15. Perturbation amplitudes with Si targets are consistently higher than SS, indicating that the perturbations increase with increasing SEY of the target. 
a)
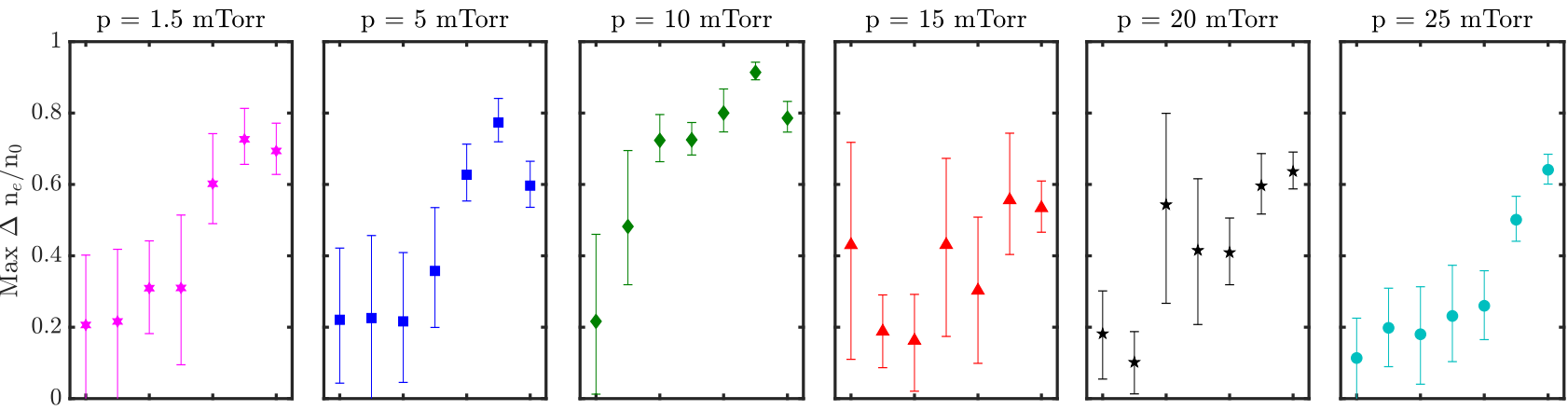

b)
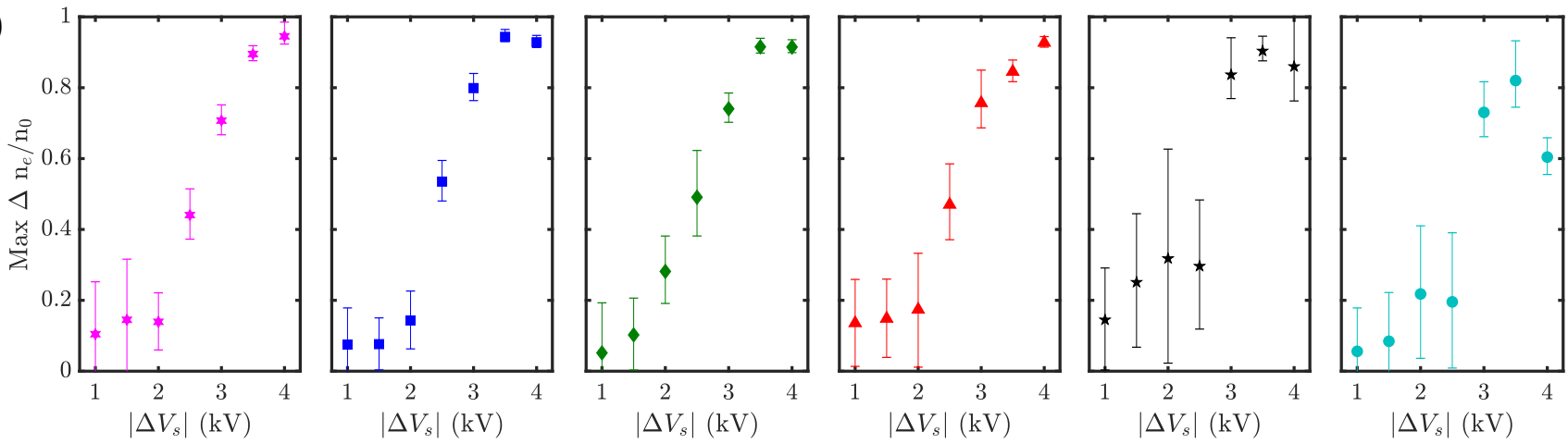

FIG. 10. Maximum perturbation in density with respect to pre-pulse values vs $\left|\Delta V_{s}\right|$ measured a) $21.7 \mathrm{~cm}$ from the target and b) $8.7 \mathrm{~cm}$ from the stand. The measurements were taken at $120 \mathrm{~W}$ input power. Note the clear departure from pre-pulse values $\gtrsim 2.0 \mathrm{kV}$.

a)

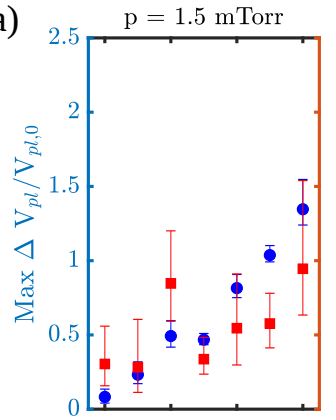

b)

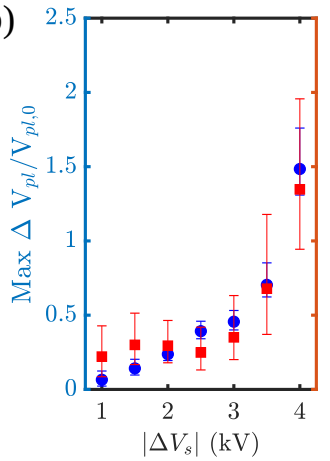

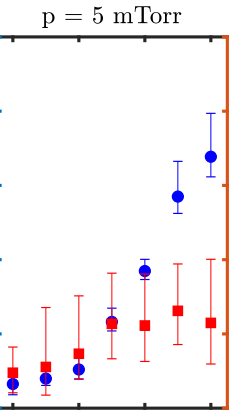

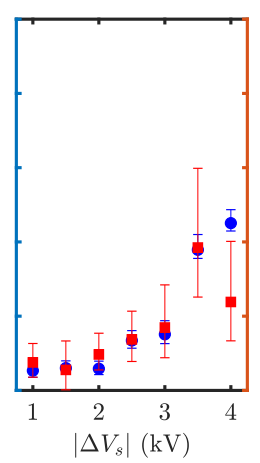

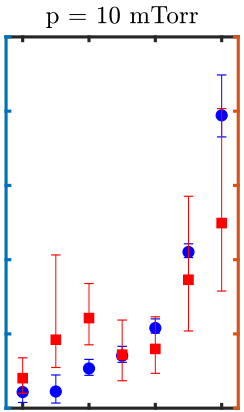

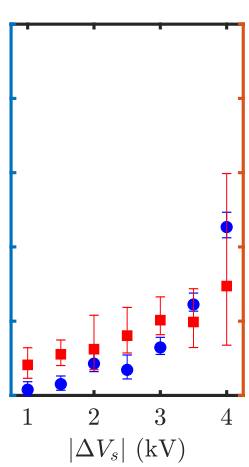

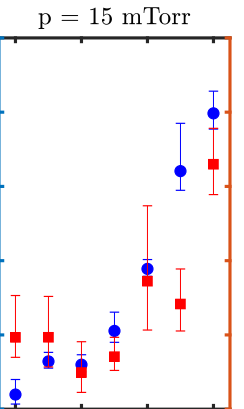
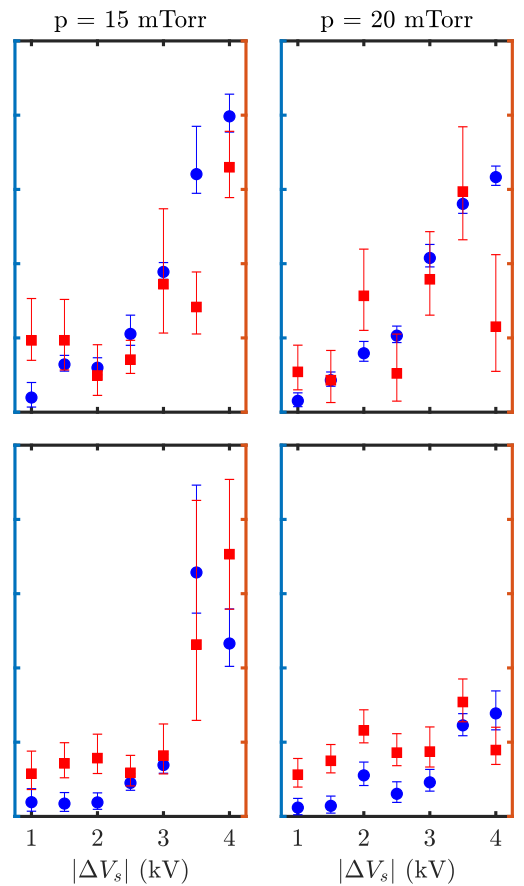
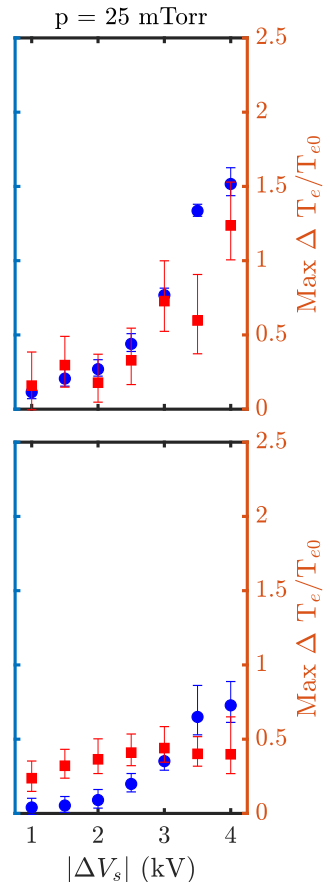

FIG. 11. Maximum perturbation in plasma potential amplitude (left axis) and electron temperature amplitude (right axis) vs $\left|\Delta V_{s}\right|$ measured a) $21.7 \mathrm{~cm}$ from the target and b) $8.7 \mathrm{~cm}$ from the stand. These measurements were also taken at $120 \mathrm{~W}$ input power. The perturbation is clearest $\gtrsim 2.0 \mathrm{kV}$. Note that perturbations in electron temperature are marginal compared to density and plasma potential. 

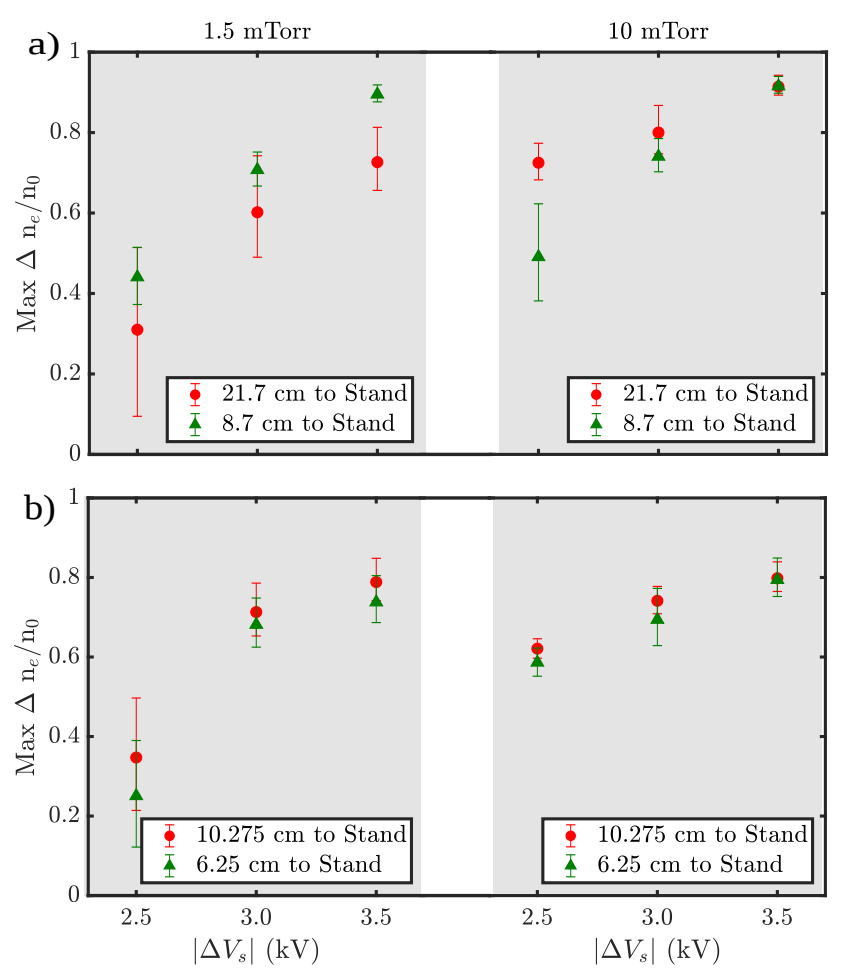

FIG. 12. The difference in perturbation amplitudes detected by a) the probes being vertically separated (the "vertical configuration") and b) the probes at the same height (the "horizontal configuration"). Measurements were taken at $120 \mathrm{~W}$ input power.

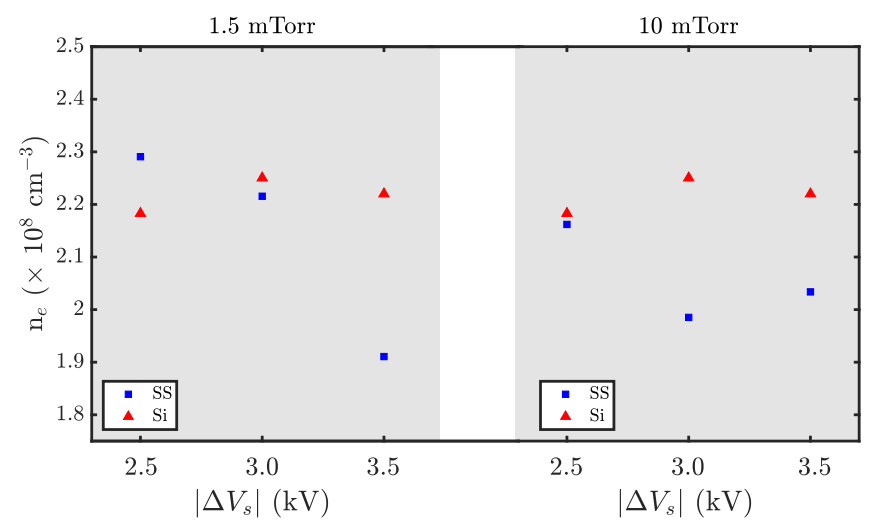

FIG. 13. The secondary electron density during the NPHV pulse, with an input power of $120 \mathrm{~W}$. These values are calculated from fast secondary electron slopes in the transition region detected in the IV curves during the voltage pulse. Errorbars are not shown to improve the visibility of the difference in density values, however it is consistently around $300 \%$ due to the low current values of the high energy electron tail of the IV characteristic. This results in values ranging from roughly $0.2 \times 10^{8}$ to $10.0 \times 10^{8} \mathrm{~cm}^{-3}$.

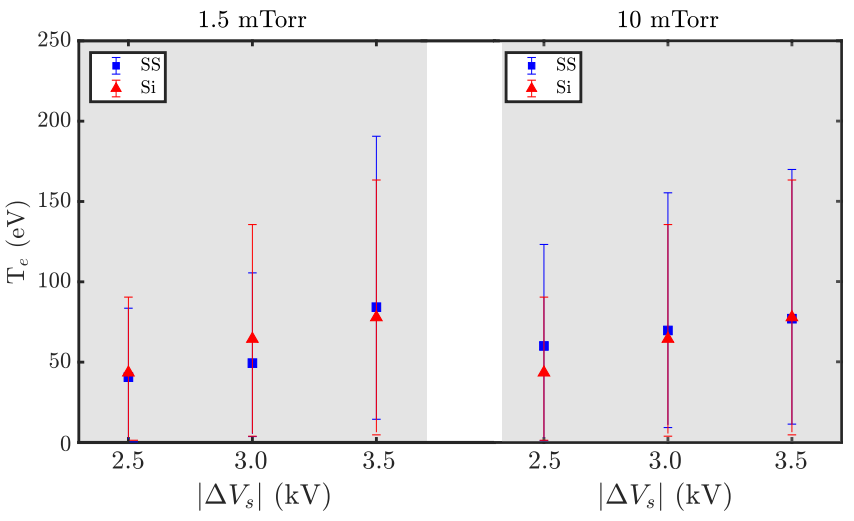

FIG. 14. The maximum electron temperature $(\mathrm{eV})$ of the secondary electrons measured during the NPHV pulse, with an input power of $120 \mathrm{~W}$.
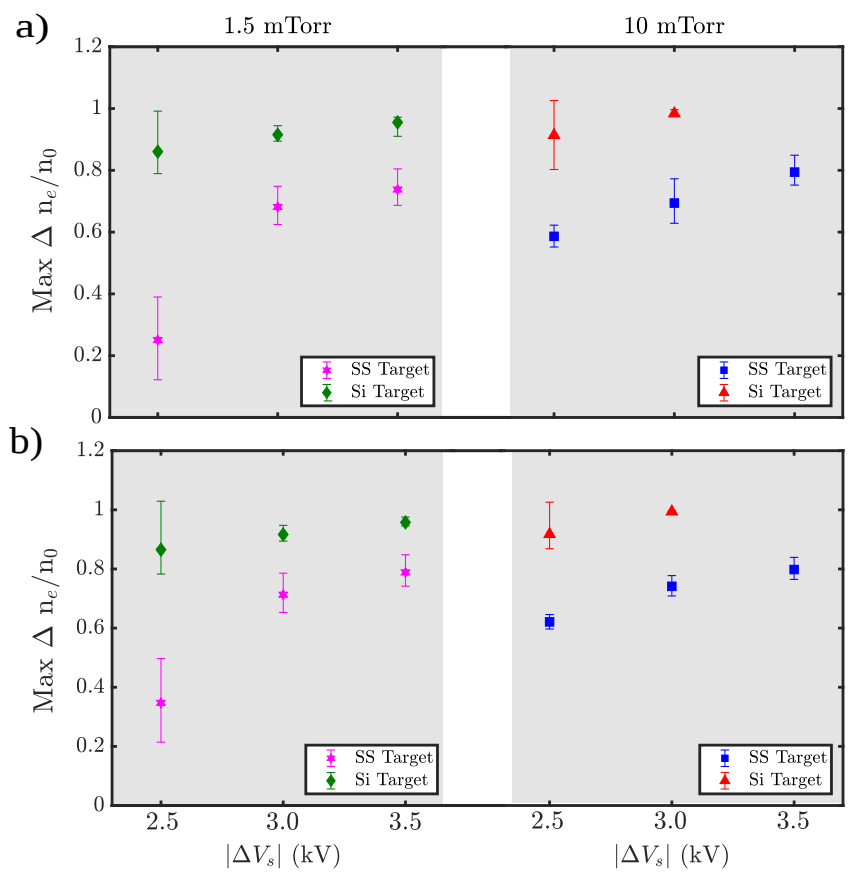

FIG. 15. The perturbation amplitude in electron density in the horizontal configuration, measured a) $6.25 \mathrm{~cm}$ from the target and b) $10.275 \mathrm{~cm}$ from the target with a stainless steel (SS) and silicon $(\mathrm{Si})$ target. Data points for $\mathrm{Si}$ at $3.5 \mathrm{kV}$ in graph b) are neglected due to erroneously high error.

\section{DISCUSSION}

\section{A. Perturbation Analysis}

The perturbations detected by the probes are not direct measurements of the plasma depletion resulting from the dynamic NPHV sheath near the pulsing target, but are rather secondary effects manifesting as delayed reactions (i.e. a propagating disturbance) in the bulk plasma. The lowest pressure tested in this experiment, 1.5 mTorr, corresponds to the lowest density and highest tempera- 
ture, as seen in Figs. 4 and 5 . These conditions correspond to the longest Child law sheath $S_{C} \approx 3.1 \mathrm{~cm}$ at the pulsing target. Therefore, the probe closest to the target at a distance of $8.7 \mathrm{~cm}$ does not measure sheath modification. Furthermore, the perturbation peaks occur roughly $5 \mu$ s after the end of the pulse, as seen in Fig. 6. This implies that the perturbations detected are not effects occurring during the voltage pulse. Figs. 7. 8 and 9 indicate that the perturbations are predominantly associated with changes in density that result in charge separation and ultimately potential fluctuations, rather than electron temperature fluctuations. The reduced perturbation amplitude associated with increased source power indicates that higher power better compensates increased disturbances to bulk plasma equilibrium.

Dependence on the proximity to the RF coil is most clearly seen in Fig. 10, where, except at 10 mTorr, the maximum perturbation amplitudes induced by $\Delta V_{s} \gtrsim 2.0$ $\mathrm{kV}$ measured $21.7 \mathrm{~cm}$ from the target are lower than their counterparts measured $8.7 \mathrm{~cm}$ from the target. Furthermore, at $21.7 \mathrm{~cm}$, the trends in perturbation amplitude with respect to NPHV pulse amplitude are less clear, particularly at higher pressures, compared to those measured at $8.7 \mathrm{~cm}$. Since the density is higher and increases more with increased pressure at $21.7 \mathrm{~cm}$ than at $8.7 \mathrm{~cm}$ (see Fig. 4), it implies that with significant increases in density (i.e. increases in power and decreased distance from $\mathrm{RF}$ coil) comes a decrease in the impact that the NPHV pulse will make.

These conditions are isolated for further analysis in Fig. 12 which details perturbation trends when the probes are positioned vertically with increasing distance from the RF coil (Fig. 12 a)) and when the probes are aligned horizontally and equally distant from the RF coil (Fig. 12 b)). In the vertical configuration, there is a bigger difference in perturbation amplitude values in the top and bottom positions, corresponding to the difference in density values measured at these positions. In the horizontal configuration, local density values change equally for both probes with increased pressure. Perturbations are overall lower, there is less dependence on pressure, and less dependence on distance to the pulsing target. This suggests that when close to the RF coil, the perturbations are more quickly compensated for by the ionizing source, and the effects of the perturbation are dampened.

\section{B. Physical Mechanism of the Perturbation}

\section{Eliminating Electron-Impact Ionization}

There are a number of preliminary explanations that can immediately be discounted in attempting to describe the phenomena observed. First of all, direct electronimpact ionization is inadequate as a mechanism to explain the observed plasma density enhancement effects seen both in the experiments reported herein (see e.g. Fig. 10 as well as in previous works (e.g. Ref. [16]).
The value of the electron-impact ionization collision rate $\nu_{e i c}=n_{g} \sigma_{e i c} \mathrm{v}_{e}$ (where $n_{g}$ is the gas density, $\sigma_{e i c}$ is the electron-impact ionization cross section, and $\mathrm{v}_{e}$ is the electron velocity) is simply not large enough at the mTorr operating pressures used here to explain the measured plasma density increases. In addition we observe that the plasma density enhancement increases with NPHV pulse voltage magnitude $\left|\Delta V_{s}\right|$ (this effect was also reported in Ref. [16]) while $\sigma_{\text {eic }}$ fall off rapidly for electron energies above $100 \mathrm{eV}$; therefore direct electron-impact ionization of neutral gas by energetic secondary electrons accelerated back through the sheath is an insignificant source of additional ionization for the $\mathrm{keV}$ electron energies encountered in PIII systems. Thus we must seek another mechanism for the observed plasma density enhancement. Similar plasma parameter perturbations were observed in low-temperature plasmas by Cluggish, et al., who suggested that their underlying mechanism was a beam-plasma instability 32 . Thus, we shall now explore this as a possible physical explanation by addressing its distinct characteristics and how they relate to the phenomena observed in these measurements.

\section{Beam-Plasma Instability}

A beam-plasma instability occurs when a beam of high energy electrons streams into a plasma, transfers energy to thermal electrons via Landau damping by exciting electron plasma waves, thereby ionizing the neutral gas and increasing the plasma density, otherwise known as plasma enhancement 32. This instability rapidly removes energy from the primary beam and can be used as e.g. the basis for a klystron-style RF amplifier [41. In a PIII system the secondary electrons ejected from the ionbombarded target form an energetic electron beam which is accelerated back into the bulk plasma; thus the conditions for the onset of a beam-plasma instability might be met as suggested in Ref. 32.

Further evidence of this instability is the increase of the beam density fraction $\alpha=\frac{n_{b}}{n_{0}}$ (where $n_{b}$ is the secondary electron beam density and $n_{0}$ is the plasma density) which leads to the increase of the instability growth rate. Since $n_{b}=\frac{j_{b}}{e \mathrm{v}_{b}}=\frac{\gamma_{s e} j_{i o n}}{e^{e} v_{b}}$ (where $j_{b}$ is the secondary electron beam density, $j_{\text {ion }}$ is the ion current density and $v_{b}=\sqrt{2 e\left|\Delta V_{s}\right| / m_{e}}$ is the secondary electron beam velocity), we expect the positive term in the expression for growth rate to increase with increasing NPHV pulse amplitude $\left|\Delta V_{s}\right|$. This effect is somewhat mitigated because $\gamma_{s e}$ and $\mathrm{v}_{b}$ are both $\propto \sqrt{\left|\Delta V_{s}\right|}$. Nonetheless, we expect the collisionless beam-plasma instability effect to increase with increasing NPHV pulse amplitude $\left|\Delta V_{s}\right|$, with a higher threshold value at higher operating pressures to overcome the increasing effect of collisions, addressed below. This argument is consistent with the trends seen in Fig. 10 as well as in other data presented in this paper. For instance, the effects of a higher SEY from silicon, implying a higher $n_{b}$ and therefore a higher $\alpha$, results 
in higher perturbation amplitudes seen in Fig. 15, thus confirming the perturbation amplitude's positive dependence on $\alpha$.

Sydorenko et al. (along with previous authors, e.g. Singhaus 42]), have found that non-ionizing electronneutral collisions can also play a role in suppressing the beam-plasma instability. Using a fluid model, Sydorenko et al. find the following expression for the growth rate of the beam-plasma instability in the absence of collisions (no collision= "ncl" case)

$$
\operatorname{Im}\left(\omega_{n c l}\right)=\omega_{p e}\left(\frac{\alpha}{13}\right) L_{n} \ln \left(L_{n}\right)\left[1-0.18 \cos \left(L_{n}+\pi / 2\right)\right]
$$

where $L_{n}=L \frac{\omega_{p}}{v_{b}}$ and $\omega_{p}$ is the plasma (i.e. Langmuir) frequency. In the presence of non-ionizing electronneutral collisions at collision rate $\nu_{e n}$, this instability growth rate is reduced to

$$
\operatorname{Im}\left(\omega_{n c l}\right)-\nu_{e n} / 2
$$

Fig. 10 shows results which are consistent with the above equation. From the data in this figure, we see that the electron beam energy, $E_{\text {beam }}=e\left|\Delta V_{s}\right|$, needed for significant plasma density enhancement increases with increasing pressure. This is because the electron-neutral collision rate $\nu_{e n}=n_{g} \sigma_{e n} \mathrm{v}_{b}$ increases linearly with target density (= neutral gas density $n_{g}=P / k_{B} T_{g}$ ); thus the instability growth rate is in general reduced at higher pressures, and may drop below the threshold needed to produce an observable plasma enhancement effect. Figs. 10 and 11 also show that there is a threshold value of NPHV pulse amplitude required for a significant perturbation amplitude, which corresponds to the minimum beam energy required.

Further evidence of this beam-plasma instability induced enhancement is visible in the temporal evolution of plasma parameters shown in Fig. 16. The figure first shows undisturbed pre-pulse parameters, followed by slight changes in densities and plasma potential during the pulse. These are a result of changing potential boundary conditions for the plasma column. Then it shows a peak in ion density corresponding to the plasma enhancement from secondary electrons, seen in the middle graph, followed by a depletion in electron density, in the top graph. This propagating wave of electron depletion is similar to the rarefaction wave measured by Chung et al. 28, 30. The peak in plasma potential corresponds to the point of greatest difference between ion density enhancement and electron density depletion.

The effects from the increased ionization from the secondary electrons is clearly seen in the ion density. The electron density, on the other hand, only undergoes minor fluctuations at the same time, seen in the upper probe measurements of Fig. 16 a). While electron and ions are created in near equal pairs from ionization, the lack of a commensurate electron density peak may be attributed to the difference in diffusion time. After the NPHV pulse ends, the resulting positive space charge from the electron-depleted region originating near the target creates a large ambipolar electric field. Due to higher mobility, the electrons created from ionization diffuse out of the region on a time scale of $\sim 0.3 \mu \mathrm{s}$. Conversely, the ions diffuse on the order of $\sim 1 \mathrm{~ms}$, and thus are more easily detected. Furthermore, the LP's RF compensation is designed to severely reduce signals at and above $10 \mathrm{MHz}$, so fast electron density enhancement will not be detected by the probe. Then, the probes measure a significant reduction in electron density as the plasma redistributes itself to recover from the pulse on the time scale of the ambipolar diffusion, $\sim 10 \mu$ s. Finally, if the perturbation is due to the beam plasma instability, the dispersion relation 43 . can be used to calculate the group velocity (propagation velocity):

$$
1=\frac{\omega_{p}^{2}}{\omega^{2}}+\frac{\omega_{p}^{2}}{\left(\omega-k \mathrm{v}_{b}\right)^{2}} \frac{n_{b}}{n_{0}} .
$$

Order of magnitude calculations of the velocity of the perturbation were made by dividing the distance between the probes in the vertical configuration, $13.0 \mathrm{~cm}$, by the time delay of the arrival of the perturbation peak between the lower and upper probes. The results are shown in Fig. 17. Order of magnitude calculations for the group velocity of equation 6 were done using values for beam density from Fig. 13 (while accounting for the wide range of error), $\mathrm{v}_{b}$ at $\left|\Delta V_{s}\right|=3.5 \mathrm{kV}$ as well as the unperturbed plasma density $n_{0}$ taken at $1.5 \mathrm{mTorr}, 120 \mathrm{~W}$. As for the wavenumber $k=\pi / L$, it is difficult to know the exact value in this bounded plasma system. However for lengths between 1 and $10 \mathrm{~cm}$, which are reasonable given the plasma chamber length, we maintain the inequality $\left|\omega / k-\mathrm{v}_{b}\right|<<\omega / k$ stipulated in Ref. [43] to use this equation. These values gave group velocity results on the order of $10^{5} \mathrm{~m} / \mathrm{s}$, which are similar to those shown in Fig. 17, thus supporting the claim that the perturbations observed propagating through the plasma after a NPHV pulse might result from a beam-plasma instability.

In summary, our data shows that perturbations in bulk plasma characteristics from NPHV pulses above $2.0 \mathrm{kV}$ cause significant deviation from conditions assumed by the Lieberman model. This therefore may result in processed materials whose implantation profile does not conform to predictions. The behaviour of the perturbations measured herein further adds to the growing body of evidence that the mechanism for the observed plasma heating and density increase in PIII plasmas may be the beam-plasma instability. This instability has a very high growth rate and thus provides an efficient mechanism for coupling energy out of the secondary electron beam and into the bulk plasma electrons. Non-ionizing electronneutral collisions can act to reduce the instability growth rate; this effect manifests itself as a higher pulse voltage being needed to observe a significant plasma density enhancement in our PIII system; analogous effects have 
a)

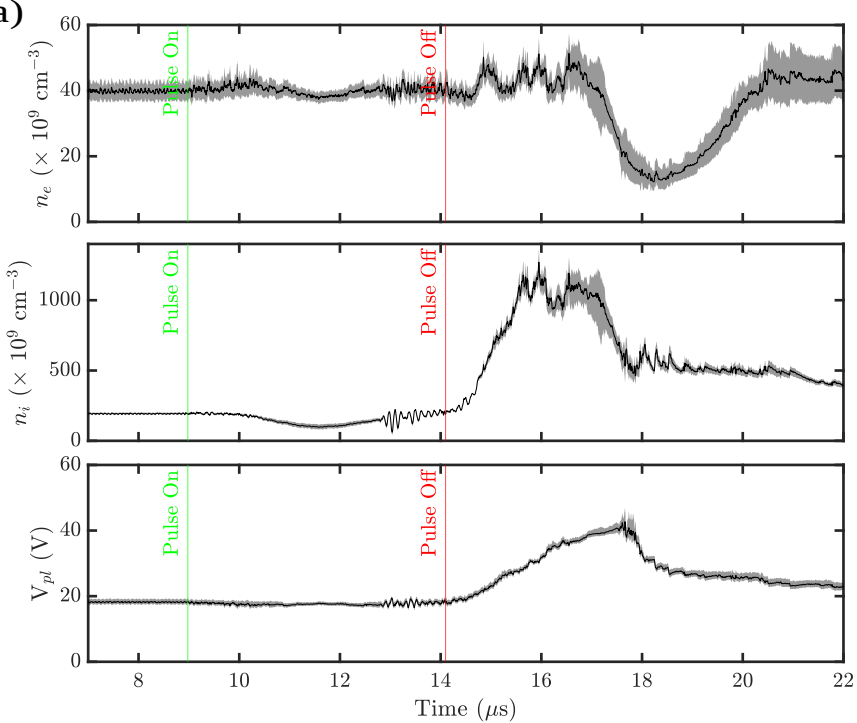

b)

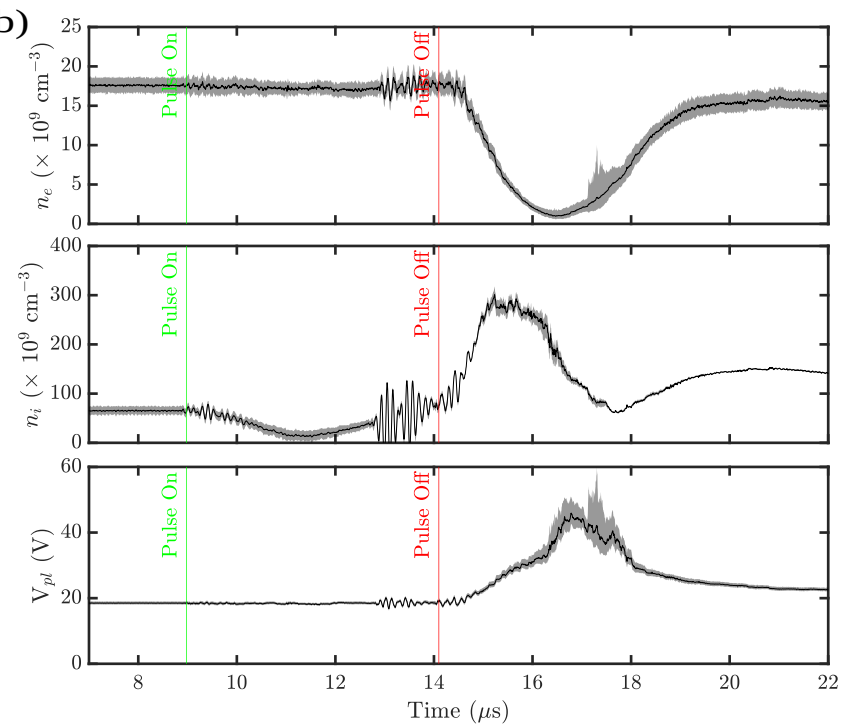

FIG. 16. LP measurements, in the vertical configuration, taken a) $21.7 \mathrm{~cm}$ from the target and b) $8.7 \mathrm{~cm}$ from the target at 120 $\mathrm{W}$ and $1.5 \mathrm{~m}$ Torr with a $4 \mathrm{kV}\left|\Delta V_{s}\right|$. The perturbations in electron and ion density correspond to a peak in plasma potential, all of which occur after the NPHV pulse. These attributes indicate a beam-plasma instability. The plasma potential tends to dip slightly during the pulse, however it is difficult to discern in this graph.

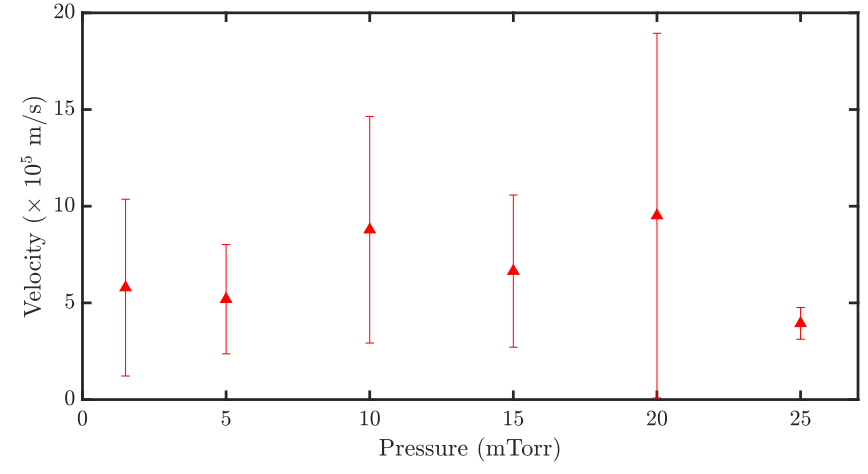

FIG. 17. Velocity values based on the time delay between perturbation peaks.

been previously reported [16].

\section{CONCLUSION}

In this article time-resolved measurements of plasma parameters - electron density, temperature and plasma potential - were performed by using RF compensated Langmuir probes installed in different locations in an inductively coupled plasma source in which a NPHV pulse was applied to a target. The probes measured perturbations as they moved from the NPHV pulsing target through the plasma. Initial analyses of the perturbations found that higher powers resulted in lower perturbation amplitudes. In particular, electron density and plasma potential were more affected by the NPHV pulse than electron temperature. Then, at fixed power and varying pressure, the perturbation amplitude was observed to grow proportionally with $\left|\Delta V_{s}\right| \gtrsim 2.0 \mathrm{kV}$, below which perturbations are negligibly above pre-pulse values. Furthermore, these results also show that the proximity from the RF coil is a major factor; at positions near the target and farther from the RF coil, pressure does not make a difference in the perturbation amplitude of electron density.

While the vertical configuration of probes is useful to see the evolution of the perturbation, there are significant complications that obscure the data. These include the non-uniform axial profile of plasma density and electron temperature since the plasma was generated at the top of the vacuum vessel away from the pulsing target. In order to study the effects of the proximity to the pulsing target on the perturbations, the probes and pulsing target are positioned in a horizontal configuration across the diameter of the chamber. Since the plasma is quasi-uniform out to $6 \mathrm{~cm}$ from the centre, this configuration minimizes the gradient effect and ensures that both probes are equidistant to the RF coil, allowing proper studies on pulsing target proximity. The results show that operating near the RF coil 1) reduces the overall amplitude of the perturbation, and 2) reduces the dependence of the amplitude on the operating pressure. This is most likely due to the ability of the plasma source to respond quicker to perturbations.

While most perturbation measurements were taken with a stainless steel target on the NPHV pulsing stand, additional measurements were performed with a silicon target. The perturbations were consistently higher when silicon was the pulsing target as opposed to stainless 
steel. Since silicon has a higher secondary electron yield coefficient, the perturbation was attributed to the effect of secondary electrons streaming into the plasma. The time evolution of electron and ion density and plasma potential showed that a peak in ion density followed by a depletion in electron density results in a spike in plasma potential.

Our results suggest a beam-plasma instability leading to plasma enhancement. The spike in ion density corresponding to increased ionization is evident in the time evolution of the densities. The measured propagation speed of this perturbation is on the same order of magnitude of the group velocity of the beam-plasma instability dispersion relation. While the results point toward a beam-plasma instability, there remains potential discrepancy between ions and electrons density evolution, such as the offset since the time of peak electron depletion and that of ion augmentation. In order to confirm the role of secondary electrons and the appearance of a beam-plasma instability leading to the observed plasma enhancement, further studies are necessary. For example, time resolved measurements of the plasma oscillations near the electron frequency would provide a more direct indication of the mechanism at hand. Combined with numerical studies, the mechanism behind the observed instability could be better understood.

In summation, the results presented in this article demonstrate that plasma conditions during PIII processing may deviate significantly from those assumed by the predictive model. For example, the bulk plasma properties lose their steady state values for $\left|\Delta V_{s}\right| \gtrsim 2.0 \mathrm{kV}$, the extent to which depends on the location relative to the pulsing stand. These results indicate that PIII processing in ICP may be improved by reorienting the pulsing target such that it is closer to the RF coil, and processing is performed parallel to the surface of the RF coil, rather than perpendicular. This has the advantage of reducing the effects of the NPHV pulse on the bulk plasma parameters, which would allow for quicker sheath recovery time and therefore faster throughput, as well as reduced disturbance to the bulk plasma parameters resulting in more spatially uniform ion deposition. Further study must be conducted to determine potential harmful effects on the target in this configuration, such as melting or arcing at higher pulsing amplitudes due to proximity to the $\mathrm{RF}$ coil.

\section{ACKNOWLEDGEMENTS}

The authors would like to thank Dr. Andrei Smolyakov for his support in interpreting the results of this experimental campaign. The authors acknowledge the support of the Natural Sciences and Engineering Research Council of Canada (NSERC), and Discovery Grants RGPIN-2015-06261 and RGPIN-2019-04333, as well as the Canada Foundation for Innovation (CFI).

\section{DATA AVAILABILITY}

The data that support the findings of this study are available from the corresponding author upon reasonable request.

\section{A. LANGMUIR PROBE SCHEMATIC}

[1] M. Shamim, J. Scheuer, R. Fetherston, and J. R. Conrad, Measurement of electron emission due to energetic ion bombardment in plasma source ion implantation, Journal of Applied Physics 70, 4756 (1991).

[2] P. Chu and C. Chan, Applications of plasma immersion ion implantation in microelectronics, Surface and Coatings Technology 136, 151 (2001).

[3] M. P. Bradley, P. R. Desautels, D. Hunter, and M. Risch, Silicon electroluminescent device production via plasma ion implantation, Physica Status Solidi (C) Current Topics in Solid State Physics 6, 6 (2009).

[4] S. Walther and R. Liebert, Development of plasma-based implant for silicon devices, Journal of Vacuum Science \& Technology B: Microelectronics and Nanometer Structures 24, 482 (2006).

[5] E. C. Jones, B. P. Linder, and N. W. Cheung, Plasma immersion ion implantation for electronic materials, Japanese Journal of Applied Physics, Part 1: Regular Papers and Short Notes and Review Papers 35, 1027 (1996).

[6] S. K. Purdy, A. P. Knights, M. P. Bradley, and G. S. Chang, Light-emitting diodes fabricated from carbon ions implanted into p-type silicon, IEEE Transactions on Electron Devices 62, 914 (2015).

[7] P. R. Desautels, M. P. Bradley, J. T. Steenkamp, and J. Mantyka, Electroluminescence in plasma ion implanted silicon, Physica Status Solidi (A) Applications and Materials Science 206, 985 (2009).

[8] C. Bortolan, C. Paternoster, S. Turgeon, C. Paoletti, M. Cabibbo, N. Lecis, and D. Mantovani, Plasmaimmersion ion implantation surface oxidation on a cobalt-chromium alloy for biomedical applications, Biointerphases 15 (2020).

[9] M. Risch and M. P. Bradley, Prospects for band gap engineering by plasma ion implantation, Physica Status Solidi (C) Current Topics in Solid State Physics 6 (2009).

[10] A. Anders, Handbook of Plasma Immersion Ion Implantation and Deposition, 1st ed. (John Wiley and Sons, Inc, 605 Third Avenue, New York, NY 10158, 2000).

[11] P. Chabert and N. Braithwaite, Physics of RadioFrequency Plasmas, 1st ed. (Cambridge University Press, The Edinburgh Building, Cambridge CB2 8RU, UK, 2011). 
a)

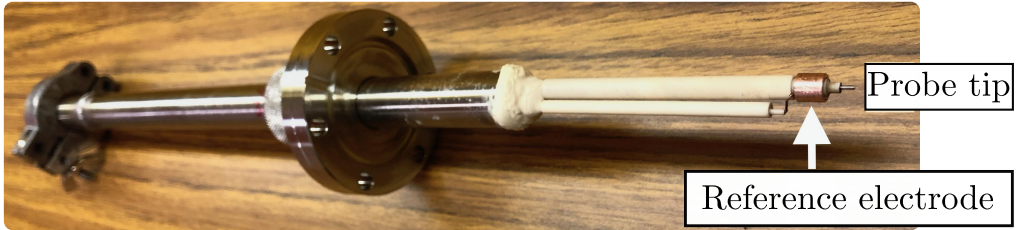

b)

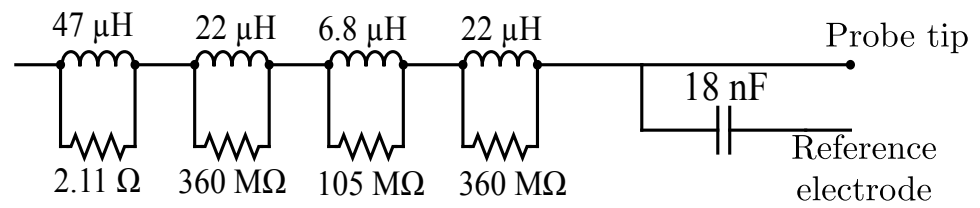

c)

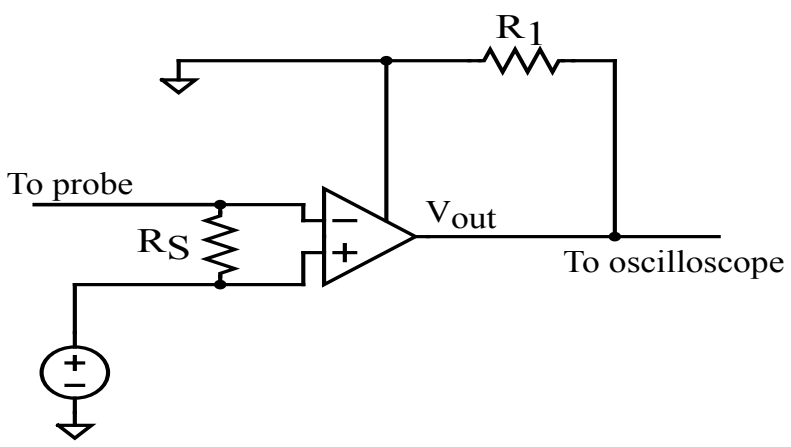

FIG. 18. a) The RF-compensated Langmuir probe used in this experiment. b) Schematic of the probe's internal circuitry, with four inductors in series to reduce the RF fluctuations on the output signal. c) Schematic of the probe's external circuitry, where $R_{1}$ is included to reduce noise in the output signal and $R_{S}$ is the sense resistor.

[12] J. R. Conrad, J. L. Radtke, R. A. Dodd, F. J. Worzala, and N. C. Tran, Plasma source ion-implantation technique for surface modification of materials, Journal of Applied Physics 62, 4591 (1987)

[13] M. Lieberman and A. Lichtenberg, Principle of Plasma Discharges and Materials Processing, 3rd ed., Vol. 1 (John Wiley and Sons, Inc, 605 Third Avenue, New York, NY 10158, 1994).

[14] J. T. Gudmundsson, N. Brenning, D. Lundin, and U. Helmersson, High power impulse magnetron sputtering discharge, Journal of Vacuum Science and Technology A: Vacuum, Surfaces, and Films 30, 030801 (2012).

[15] W. Y. L. Ling, S. Zhang, H. Fu, M. Huang, J. Quansah, $\mathrm{X}$. Liu, and N. Wang, A brief review of alternative propellants and requirements for pulsed plasma thrusters in micropropulsion applications, Chinese Journal of Aeronautics 33, 2999 (2020).

[16] S. Qin, M. P. Bradley, P. L. Kellerman, and K. Saadatmand, Measurements of secondary electron emission and plasma density enhancement for plasma exposed surfaces using an optically isolated Faraday cup, Review of Scientific Instruments 73, 1153 (2002)

[17] R. A. Stewart and M. A. Lieberman, Model of plasma immersion ion implantation for voltage pulses with finite rise and fall times, Journal of Applied Physics 70, 3481 (1991)

[18] G. A. Emmert and M. A. Henry, Numerical simulation of plasma sheath expansion, with applications to plasmasource ion implantation, Journal of Applied Physics 71, $113(1992)$
[19] B. Wood, Displacement current and multiple pulse effects in plasma source ion implantation, Journal of Applied Physics 73, 4770 (1993).

[20] D. Wang, T. Ma, and X. Deng, Model of collisional sheath evolution in plasma source ion implantation, Journal of Applied Physics 74, 2986 (1993)

[21] E. Keiter, W. Hitchon, and M. Goeckner, A kinetic model of pulsed sheaths, Physics of Plasmas 1, 3709 (1994).

[22] T. Chung, H. Yoon, and J. Lee, Scaling laws verification for capacitive rf-discharge ar plasma using particle-in-cell simulations, Journal of Applied Physics 78, 6441 (1995).

[23] X. Tian and P. Chu, Modeling of the relationship between implantation parameters and implantation dose during plasma immersion ion implantation, Physics Letters, Section A: General, Atomic and Solid State Physics 277, 42 (2000).

[24] T. Daube, P. Meyer, K.-U. Riemann, and H. Schmitz, Relaxation phenomena in pulsed discharges, Journal of Applied Physics 91 (2001).

[25] B. P. Cluggish and C. P. Munson, Sheath overlap during large scale plasma source ion implantation, IEEE International Conference on Plasma Science 84, 5937 (1998).

[26] A. Khodaee, RF-compensated Langmuir Probe Diagnostics of Pulsed Plasma Ion Implantation System, Master's thesis, University of Saskatchewan (2019).

[27] M. A. Lieberman and S. Ashida, Global models of pulsepower-modulated high-density, low-pressure discharges, Plasma Sources Science and Technology 5, 145 (1996).

[28] K.-J. Chung, Y.-S. Hwang, and G.-H. Kim, Dynamic sheath expansion in a non-uniform plasma with ion drift, Plasma Sources Science and Technology 20 (2011). 
[29] K.-J. Chung, J.-M. Choe, G.-H. Kim, and Y.-S. Hwang, Numerical investigation of plasma recovery in plasma source ion implantation, Thin Solid Films 521, 197 (2012).

[30] K.-J. Chung, B. Jung, G.-H. Kim, and Y.-S. Hwang, Experimental investigation of plasma recovery during the pulse-off time in plasma source ion implantation, Thin Solid Films 547, 13 (2013).

[31] B. Briehl and H. Urbassek, Simulation of sheath and presheath dynamics in piii, Surface and Coatings Technology 156, 131 (2002).

[32] B. P. Cluggish and C. P. Munson, Secondary electron enhanced discharges in plasma source ion implantation, Journal of Applied Physics 84, 5945 (1998).

[33] A. V. Phelps and Z. L. Petrović, Cold-cathode discharges and breakdown in argon: surface and gas phase production of secondary electrons, Plasma Sources Science and Technology 8, 10.1088/0963-0252/8/3/201 (1999).

[34] M. P. Bradley and C. J. Steenkamp, Time-resolved ion and electron current measurements in pulsed plasma sheaths, IEEE Transactions on Plasma Science 34, 1156 (2006).

[35] K. Takashi, C. Charles, R. Boswell, and R. Hatakeyama, Pulsed Electrostatic Langmuir Probe Technique for Electron Energy Distribution Function Measurement in Radio-Frequency-Driven Plasmas, Tech. Rep. 2 (The Australian National University, SP3 Department, Rsphysse, Australian National University, Mills Road,
Acton ACT 0200, 2007).

[36] F. Chen, Lecture notes on langmuir probe diagnostics, http://www. seas.ucla.edu/ ffchen/Publs/ Chen210R.pdf (2003).

[37] P. A. Chatterton, J. A. Rees, W. L. Wu, and K. AlAssadi, A self-compensating langmuir probe for use in $\mathrm{rf}$ (13.56 mhz) plasma systems, Vacuum 42, 489 (1991).

[38] Y. Sukhanov, A. Ershov, K. Rudenko, and A. Orlikovsky, Comparative study of inductively coupled and microwave bf3 plasmas for microelectronic technology applications, Proceedings of SPIE - The International Society for Optical Engineering 5401 (2004)

[39] V. A. Godyak, R. B. Piejak, and B. M. Alexandrovich, Electron energy distribution function measurements and plasma parameters in inductively coupled argon plasma, Plasma Sources Science and Technology 11, 525 (2002).

[40] F. Chen, Introduction to Plasma Physics and Controlled Fusion (Plenum Press, New York, 1984).

[41] H. S. Uhm, Relativistic plasma klystron amplifier in connection with application to high gradient accelerators, Proceedings of the IEEE Particle Accelerator Conference 4, 2678 (1993)

[42] H. E. Singhaus, Beam-temperature effects on the electrostatic instability for an electron beam penetrating a plasma, Physics of Fluids 7, 1534 (1964).

[43] A. Fridman and L. Kennedy, Plasma Physics and Engineering, 2nd ed. (CRC Press, 6000 Broken Sound Parkway NW, Suite 300, Boca Raton, FL, 33487, 2011). 